\title{
Predicting the Maximum and Minimum Eigenvalues of a Random Matrix Filled with iid Normal Variates: A Study in Simulation
}

\author{
Princila, Soubhik Chakraborty ${ }^{*}$ \\ Department of Mathematics, Birla Institute of Technology Mesra, Ranchi-835215, India.
}

How to cite this paper: Princila, Soubhik Chakraborty. (2021) Predicting the Maximum and Minimum Eigenvalues of a Random Matrix Filled with iid Normal Variates: A Study in Simulation. Journal of Applied Mathematics and Computation, 5(1), 18-27. DOI: 10.26855/jamc.2021.03.003

Received: December 12, 2020

Accepted: January 5, 2021

Published: January 22, 2021

*Corresponding author: Soubhik Chakraborty, Department of Mathematics, Birla Institute of Technology Mesra, Ranchi-835215, India.

Email: soubhikc@yahoo.co.in

\begin{abstract}
This paper involves a simulation study to predict the maximum and minimum eigenvalues of a random matrix whose elements are coming from $N\left(\mu, \sigma^{2}\right)$. In the first study, we fix $\sigma$ and for different values of $\mu$ we generate 100 matrices of order $10 \times 10$ in MATLAB. Then, by plotting a graph between mean maximum eigenvalue and $\mu$, a pattern is detected and we obtain the equation of best curve fit using MS-EXCEL. However, no pattern is detected for mean minimum eigenvalue with respect to $\mu$. In the second study, the same procedure is repeated except that here we fix $\mu$ and vary $\sigma$. Here the reverse happens interestingly. Pattern is detected for mean minimum eigenvalue with respect to $\sigma$ but no pattern is detected for the case of mean maximum eigenvalue. Both these studies are repeated for random matrices of order $5 \times 5$ with identical results as in the case of $10 \times 10$ matrices except that the magnitude of the maximum eigenvalue is reduced by about half when the order of the matrices is reduced by half while magnitude of the minimum eigenvalue is not significantly affected. The paper also includes a theoretical analysis of predicting the range of the sum of all the eigenvalues of a diagonalizable random matrix with the help of its trace and Chebyshev's inequality. This paper is organised as follows. Section 1 is the introduction. Section 2 is the literature review. Section 3 gives the methodology. Section 4 gives the experimental results and discussion. Section 5 provides some theoretical results for a diagonalizable random square matrix. Finally, Section 6 gives the concluding remarks.
\end{abstract}

\section{Keywords}

Random matrix, Normal distribution, Eigenvalues, Trace, Chebyshev's inequality, Simulation, Curve fitting

\section{Introduction}

A matrix is not just an array of numbers but a representation of a linear transformation. Every square matrix has eigenvalues. In particular, eigenvalues are a special set of scalars associated with a linear system of equations (i.e., a matrix equation) that are sometimes also known as characteristic roots or latent roots [1].

Let $A$ be a square matrix of order $n \times n$, then a number (real or complex) $\lambda$ is said to be eigenvalue of matrix $A$ if there exists a column matrix $\mathrm{X}$ of order $\mathrm{n} \times 1$ such that,

where,

$$
\mathrm{AX}=\lambda \mathrm{X}
$$


$\lambda=$ eigenvalue of matrix A.

$\mathrm{X}=$ eigenvector of matrix A.

Eigenvalues and their corresponding eigenvectors give us important information about the linear transformation. Initially, eigenvalues and eigenvectors were used to study the principle axis of the rotational motion of rigid bodies; they now have several applications such as in vibration and stability analysis, atomic orbitals, facial recognition and matrix diagonalization. One of the very interesting properties of eigenvalues is that the sum of the eigenvalues of a diagonalizable square matrix is equal to its trace (sum of its diagonal elements) i.e., for a square matrix of order nxn,

where,

$$
\operatorname{tr}(A)=\lambda_{1}+\lambda_{2}+\lambda_{3}+\ldots \ldots+\lambda_{\mathrm{n}}=\sum_{i=1}^{n} \lambda_{\mathrm{i}}
$$

$\mathrm{A}=$ diagonalizable square matrix.

$\lambda$ 's $=$ eigenvalues of $\mathrm{A}$.

In this paper, we build random matrices filled with iid (independently and identically distributed) normal variates.

A random variable $\mathrm{X}$ is said to follow normal distribution if its p.d.f. is given by:

$$
\mathrm{f}(\mathrm{x})=\frac{1}{\sigma \sqrt{2 \pi}} e^{\frac{-1}{2}\left(\frac{x-\mu}{\sigma}\right)^{2}},-\infty<\mathrm{x}<\infty
$$

with $-\infty<\mu<\infty$ and $\sigma>0$. Here $\mu$, the population mean and $\sigma^{2}$, the population variance, are the parameters of the distribution [2].

To generate normal variates, we are using the Box Muller transformation [3] as used in Monte Carlo Simulation (a tacit combination of probability theory and sampling technique). We store these random variates in the form of a matrix of order $10 \times 10$.

\section{Literature Review}

A matrix in which elements are random variables is known as random matrix. We conducted our work on random matrices whose elements are coming from normal distribution. As mentioned earlier, every square matrix has eigenvalues, a special set of scalars associated with a linear system of equations (i.e., a matrix equation) that are sometimes also known as characteristic roots, latent roots [1]. Eigenvalues of a random matrix follow a particular probability distribution [4]. In fact, it is the work in [4] illustrating the distribution of eigenvalues and eigenvectors which drew our interest towards the topic. The fluctuations of the maximum eigenvalue for $n \times n$ random matrices in the limit of large $n$ have been studied in [5]. The statistical behaviour of eigenvalues for real symmetric random matrices filled with iid (independently and identically distributed) Gaussian variates has been studied in [6].

\section{Methodology}

In the first study, we fix the parameter $\sigma$ at 5 and vary the parameter $\mu$ in the range of 5-50 and at each $\mu$ we generate 100 matrices of order $10 \times 10$ and obtain mean of maximum eigenvalue of each of the 100 matrices using MATLAB. Next, we represent this data spread by assigning a 'best fit' regression line along the entire range of 5-50 by the least square method. For this, we plot the graph between parameter ' $\mu$ ' along abscissa and the 'mean of maximum eigenvalue' along ordinate. Using MS-EXCEL, the equation of best fit is obtained. This procedure is repeated to obtain the line of best fit between parameter $\mu$ and mean of minimum eigenvalue.

In the second study, we fix the parameter $\mu$ at 10 and vary $\sigma$ from 5-14 and the same procedure is followed to obtain the mean value of maximum eigenvalue of 100 random matrices. The regression equation for best fit is obtained as in the first study. The same procedure is repeated for the minimum eigenvalue case.

In addition to these studies, we also provide a theoretical analysis of predicting the range of the sum of all the eigenvalues of a diagonalizable random matrix with the help of its trace and Chebyshev's inequality [2].

\section{Experimental Results and Discussion}

With the help of MATLAB, we generated an algorithm to obtain the mean of maximum and minimum eigenvalue at different ' $\mu$ ' in the range 5-50. The experimental results are tabulated in Table 1.

Figure 1 gives the line of best fit between parameter $\mu$ and mean of maximum eigenvalue of a random matrix of order $10 \times 10$. In this graph, we can observe that the coefficient of determination $R^{2}$ is 0.9996 which is close to 1 which means the error sum of square is negligible and hence a straight line will give the best fit for predicting the maximum eigenvalue $\mathrm{y}$ for a given $\mu$ ( $\mathrm{x}$ in Figure 1).

Similarly, for mean minimum eigenvalue, we have the following data tabulated in Table 2. 
Table 1. Mean Maximum Eigenvalue versus $\mu$ for $10 \times 10$ random matrix

\begin{tabular}{cccc}
\hline Parameter $\mu$ & Mean Maximum Eigenvalue $(\mathrm{m})$ & Standard Deviation (S.D) & $(\mathrm{m}-3 \times$ S.D, m+3×S.D) \\
\hline 5 & 59.2511 & 11.7681 & $(23.9468,94.5554)$ \\
10 & 99.9690 & 17.7796 & $(46.6302,153.3078)$ \\
15 & 148.3799 & 16.2737 & $(99.5588,197.201)$ \\
20 & 198.7490 & 15.8004 & $(151.3478,246.1502)$ \\
25 & 253.2702 & 16.7780 & $(202.9362,303.6042)$ \\
30 & 299.4396 & 15.5603 & $(252.7587,346.1205)$ \\
35 & 351.3702 & 16.6412 & $(301.4466,401.2938)$ \\
40 & 402.5232 & 17.3116 & $(350.5884,454.458)$ \\
50 & 452.3266 & 17.0785 & $(401.0911,503.5621)$ \\
\end{tabular}

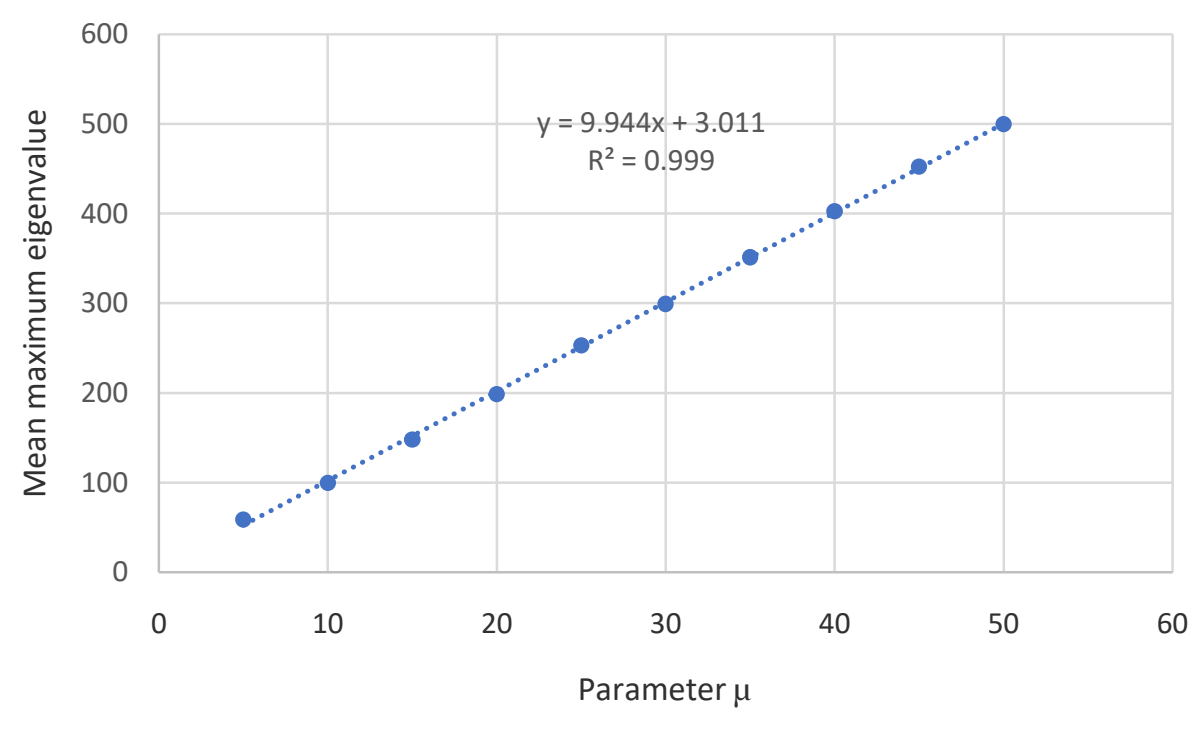

Figure 1. Line of best fit between parameter $\mu$ and mean maximum eigenvalue of $10 \times 10$ random matrix.

Table 2. Mean Minimum Eigenvalue versus parameter $\mu$ for $10 \times 10$ random matrix

\begin{tabular}{cccc}
\hline Parameter $\mu$ & Mean Minimum Eigenvalue $(\mathrm{m})$ & Standard Deviation (S.D) & $(\mathrm{m}-3 \times$ S.D, m+3×S.D) \\
\hline 5 & 9.6402 & 6.3162 & $(-9.3084,28.5888)$ \\
10 & 10.0280 & 6.3972 & $(-9.1636,29.2196)$ \\
15 & 9.3096 & 6.5318 & $(-10.2858,28.905)$ \\
20 & 10.1892 & 6.7182 & $(-9.9654,30.3438)$ \\
25 & 10.7941 & 6.2892 & $(-8.0735,29.6617)$ \\
30 & 9.3905 & 6.5460 & $(-10.2475,29.0285)$ \\
35 & 9.7242 & 5.5570 & $(-6.9468,26.3952)$ \\
40 & 10.0603 & 6.0227 & $(-8.0078,28.1284)$ \\
45 & 10.5186 & 5.4257 & $(-5.7585,26.7957)$ \\
50 & 10.9682 & 6.0937 & $(-7.3129,29.2493)$ \\
\hline
\end{tabular}




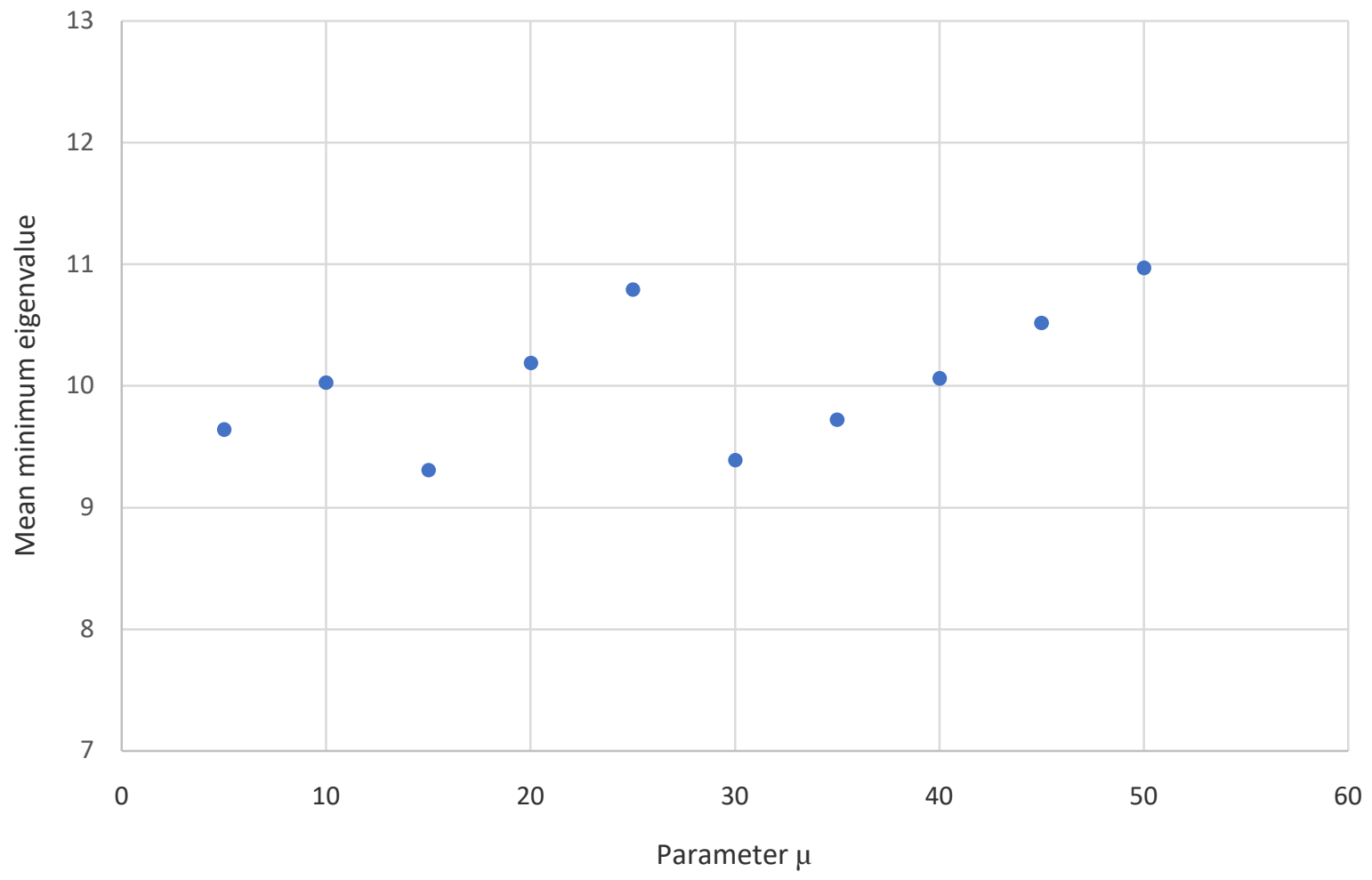

Figure 2. Line of best fit between $\mu$ and mean minimum eigenvalue of $10 \times 10$ random matrix.

Applying run test of randomness [2] [3] using MATLAB to the data of mean minimum eigenvalue in Table 2, it was found that the sequence of mean minimum eigenvalue is random and therefore there is no dependence on the parameter $\mu$ of the input distribution.

Now we predict the maximum and minimum eigenvalue of matrix for fixed $\mu=10$ and different $\sigma$. For that, we have experimental data tabulated in Table 3 and Table 4 respectively.

Again, applying run test of randomness using MATLAB to the data of mean maximum eigenvalue in Table 3, it was found that the sequence of mean maximum eigenvalue is random and therefore there is no dependence on the parameter $\sigma$ of the input distribution.

Table 3. Mean Maximum Eigenvalue versus $\sigma$ for $10 \times 10$ random matrix

\begin{tabular}{cccc}
\hline Parameter $\sigma$ & Mean Maximum Eigenvalue $(\mathrm{m})$ & Standard Deviation (S.D) & $(\mathrm{m}-3 \times$ S.D, m+3×S.D) \\
\hline 5 & 99.5809 & 4.8845 & $(85.0474,114.1144)$ \\
6 & 99.9720 & 6.2665 & $(81.1725,118.7715)$ \\
7 & 100.3167 & 81900 & $(75.7467,124.8867)$ \\
8 & 99.3512 & 8.2630 & $(74.5622,124.1402)$ \\
9 & 99.9021 & 8.1212 & $(75.5385,124.2657)$ \\
10 & 100.0331 & 8.9047 & $(73.319,126.7472)$ \\
11 & 100.2392 & 10.1799 & $(69.6995,130.7789)$ \\
12 & 99.5427 & 13.5585 & $(58.8672,140.2182)$ \\
13 & 98.4264 & 13.9355 & $(86.6199,110.2329)$ \\
14 & 99.2643 & 19.0614 & $(42.0801,156.4485)$ \\
\hline
\end{tabular}




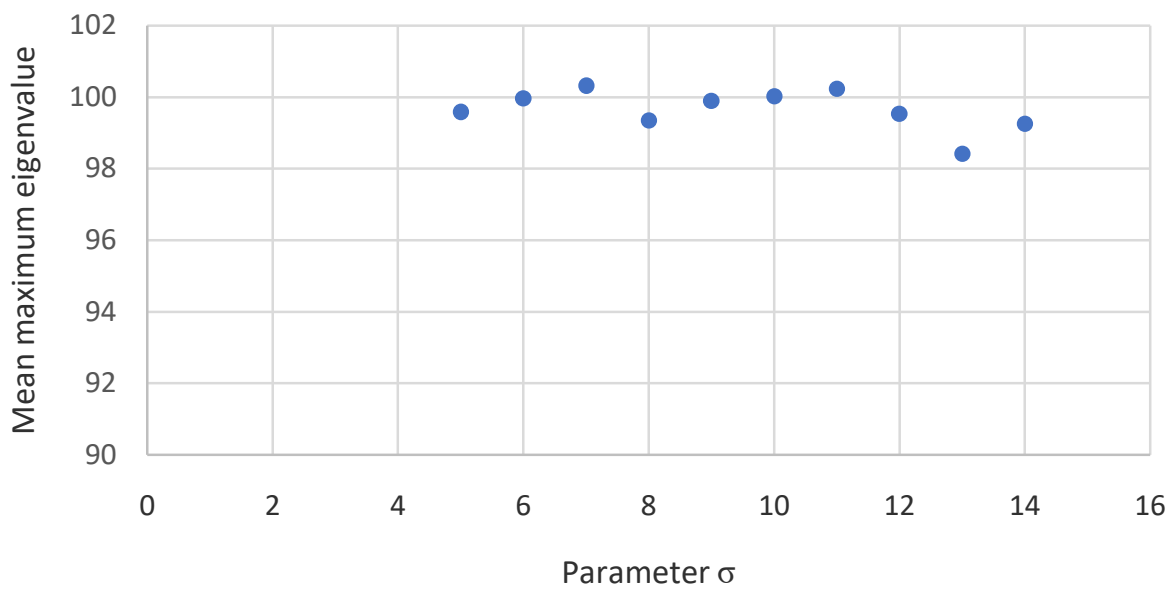

Figure 3. Line of best fit between $\sigma$ and mean maximum eigenvalue of $10 \times 10$ random matrix.

Table 4. Mean Minimum Eigenvalue versus $\sigma$ for $10 \times 10$ random matrix

\begin{tabular}{cccc}
\hline Parameter $\sigma$ & Mean Minimum Eigenvalue $(\mathrm{m})$ & Standard Deviation (S.D) & $(\mathrm{m}-3 \times$ S.D, m+3×S.D) \\
\hline 5 & 3.5306 & 2.1159 & $(-2.8117,9.8837)$ \\
6 & 3.9492 & 2.3673 & $(-3.1527,11.0511)$ \\
7 & 4.5236 & 2.8924 & $(-4.1536,13.2008)$ \\
8 & 5.2178 & 3.1681 & $(-4.2865,14.7221)$ \\
9 & 6.1168 & 3.9395 & $(-5.7017,17.9353)$ \\
10 & 6.5245 & 3.9824 & $(-5.4227,18.4717)$ \\
11 & 7.6441 & 4.6423 & $(-6.2828,21.571)$ \\
12 & 7.4451 & 4.9158 & $(-7.3023,22.1925)$ \\
14 & 7.4628 & 5.0715 & $(-7.7517,22.6773)$ \\
\hline
\end{tabular}

Line of best fit for the data of Table 4 is given in Figure 4 .

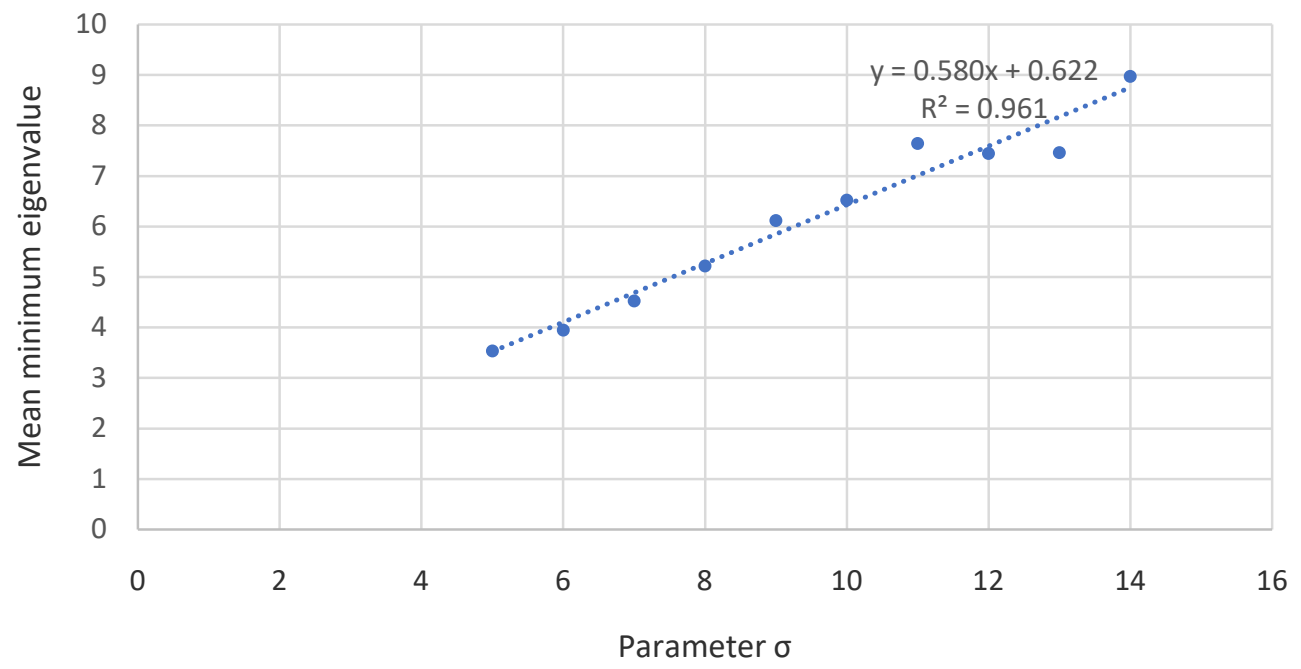

Figure 4. Line of best fit between $\sigma$ and mean minimum eigenvalue of $10 \times 10$ random matrix. 
The regression equation of straight line with $\mathrm{R}^{2}=0.9617$ is given as:

$$
y=0.5807 x+0.6223
$$

Where $\mathrm{y}$ is the minimum eigenvalue and $\mathrm{x}$ in Figure 4 is the parameter $\sigma$. Clearly there is an approximate linear pattern.

Now we wish to see the effect of reducing the order of the matrix on predicted maximum and minimum eigenvalues. For this, we performed the same experiment as done earlier taking random matrices of order $5 \times 5$ filled with normal variates. Our results are summarized in Table 5 to Table 8 and corresponding Figure 5 to Figure 8.

Table 5. Mean Maximum Eigenvalue versus $\mu$ for $5 \times 5$ random matrix

\begin{tabular}{cccc}
\hline Parameter $\mu$ & Mean Maximum Eigenvalue $(\mathrm{m})$ & Standard Deviation (S.D) & $(\mathrm{m}-3 \times$ S.D, m+3×S.D) \\
\hline 5 & 39.0180 & 9.5340 & $(10.416,67.62)$ \\
10 & 53.5850 & 14.4913 & $(10.1111,97.0589)$ \\
15 & 75.7891 & 16.4006 & $(26.5873,124.9909)$ \\
20 & 99.1222 & 15.8784 & $(51.487,146.7574)$ \\
25 & 123.3892 & 13.5113 & $(82.8553,163.9231)$ \\
30 & 150.8866 & 16.1341 & $(102.4843,199.2889)$ \\
35 & 174.9967 & 15.6293 & $(128.1088,221.8846)$ \\
40 & 199.4199 & 13.7638 & $(158.1285,240.7113)$ \\
45 & 224.7122 & 13.9411 & $(182.8889,266.5355)$ \\
50 & 248.2515 & 16.8582 & $(197.6769,298.8261)$ \\
\hline
\end{tabular}

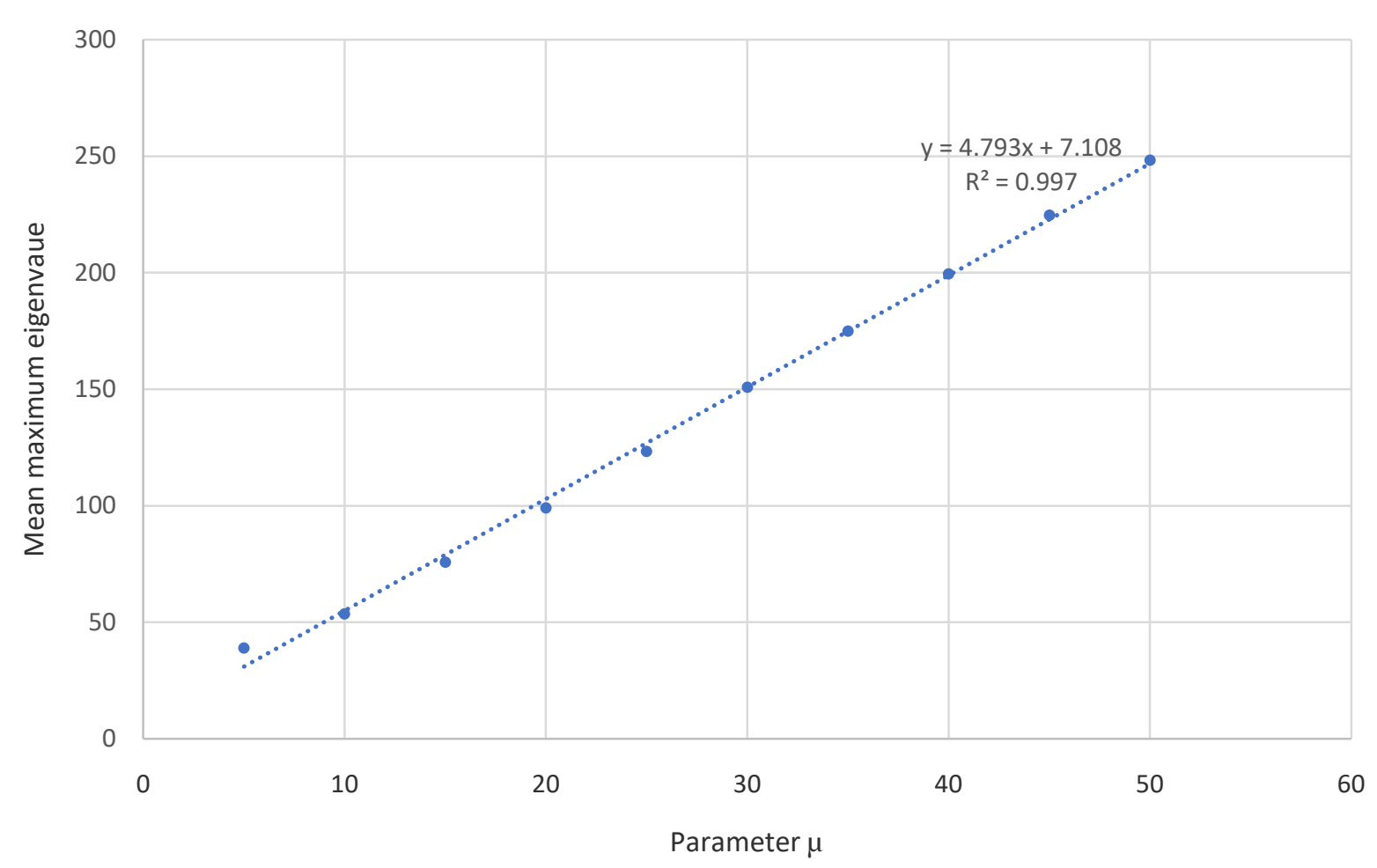

Figure 5 . Line of best fit between parameter $\mu$ and mean maximum eigenvalue of $5 \times 5$ random matrix. 
Table 6. Mean Minimum Eigenvalue versus $\mu$ of $5 \times 5$ random matrix

\begin{tabular}{cccc}
\hline Parameter $\mu$ & Mean Minimum Eigenvalue $(\mathrm{m})$ & Standard Deviation (S.D) & $(\mathrm{m}-3 \times$ S.D, m+3×S.D) \\
\hline 5 & 8.5043 & 6.2800 & $(-10.3357,27.3443)$ \\
10 & 9.9938 & 6.3722 & $(-9.1228,29.1104)$ \\
15 & 9.3874 & 5.8505 & $(-8.1641,26.9389)$ \\
20 & 10.5926 & 6.1631 & $(-7.8967,29.0819)$ \\
25 & 11.0495 & 6.6934 & $(-9.0307,31.1297)$ \\
30 & 9.9868 & 6.4685 & $(-9.4187,29.3923)$ \\
35 & 9.7861 & 6.0403 & $(-8.3348,27.907)$ \\
40 & 8.7099 & 5.4502 & $(-7.6407,25.0605)$ \\
45 & 10.0005 & 6.5538 & $(-9.6609,29.6619)$ \\
50 & 10.7457 & 6.8691 & $(-9.8616,31.353)$ \\
\hline
\end{tabular}

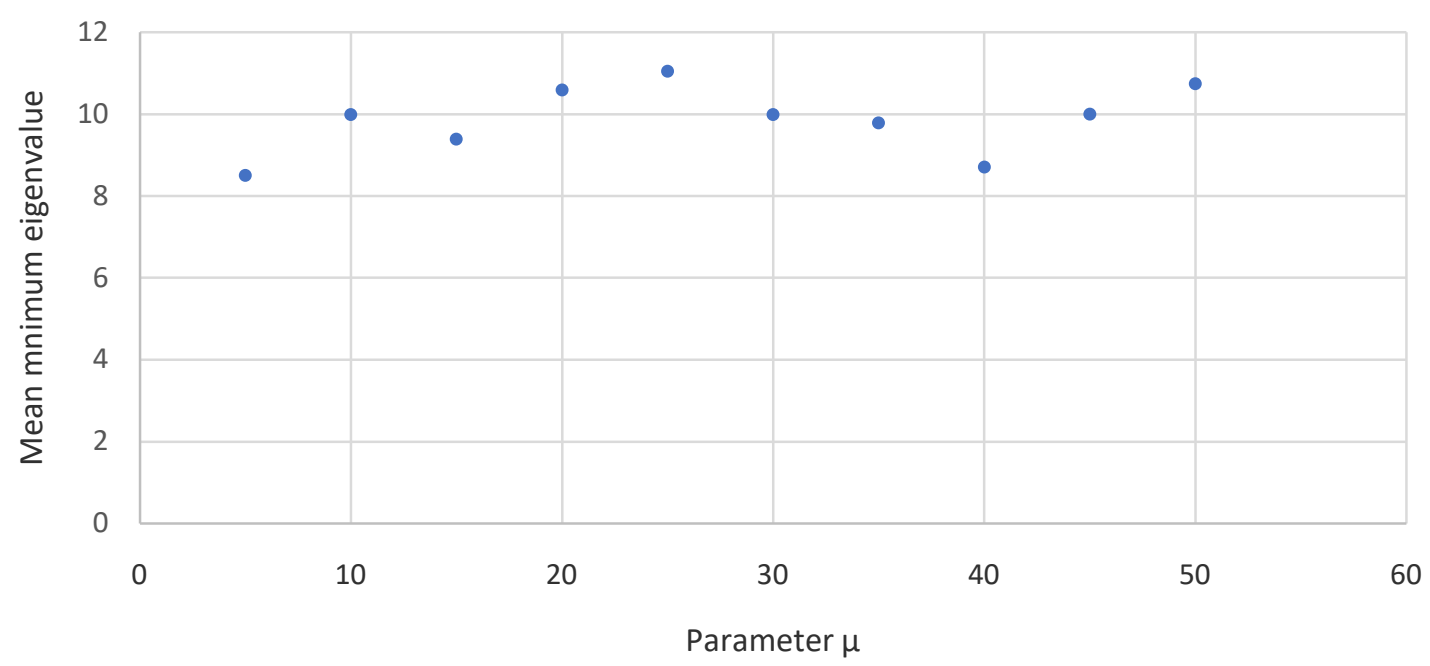

Figure 6. Line of best fit between parameter $\mu$ and mean minimum eigenvalue of $5 \times 5$ random matrix.

Table 7. Mean Maximum Eigenvalue versus $\sigma$ of $5 \times 5$ random matrix

\begin{tabular}{cccc}
\hline Parameter $\sigma$ & Mean Maximum Eigenvalue $(\mathrm{m})$ & Standard Deviation (S.D) & $(\mathrm{m}-3 \times$ S.D, m+3×S.D) \\
\hline 5 & 49.8075 & 4.8878 & $(35.1441,64.4709)$ \\
6 & 48.1233 & 6.0096 & $(30.0945,66.1521)$ \\
7 & 51.0190 & 7.2669 & $(29.2183,72.8197)$ \\
8 & 51.3009 & 8.8382 & $(24.7863,77.8155)$ \\
9 & 50.7241 & 9.7380 & $(21.5101,79.9381)$ \\
10 & 49.4567 & 11.8318 & $(13.9613,84.9521)$ \\
11 & 51.1842 & 10.5389 & $(19.5675,82.8009)$ \\
12 & 50.4408 & 11.1660 & $(16.9428,83.9388)$ \\
13 & 54.1846 & 13.8084 & $(12.7594,95.6098)$ \\
14 & 51.8752 & 14.4830 & $(8.4262,95.3242)$ \\
\hline
\end{tabular}




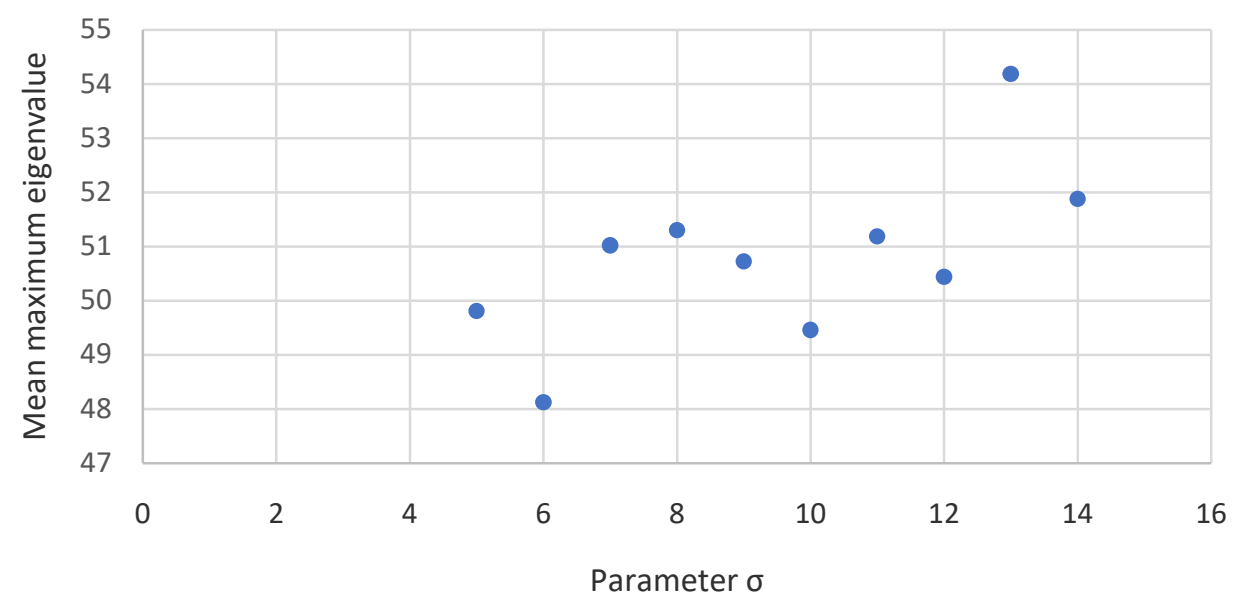

Figure 7. Line of best fit between parameter $\sigma$ and mean maximum eigenvalue of $5 \times 5$ random matrix.

Table 8. Mean Minimum Eigenvalue versus $\sigma$ of $5 \times 5$ random matrix

\begin{tabular}{cccc}
\hline Parameter $\sigma$ & Mean minimum Eigenvalue $(\mathrm{m})$ & Standard Deviation (S.D) & $(\mathrm{m}-3 \times$ S.D, m+3×S.D) \\
\hline 5 & 3.4823 & 2.2058 & $(-3.1351,10.0997)$ \\
6 & 4.3867 & 2.7450 & $(-3.8483,12.6217)$ \\
7 & 4.4096 & 3.1799 & $(-5.1301,13.9493)$ \\
8 & 4.9317 & 3.2039 & $(-4.68,14.5434)$ \\
9 & 6.3405 & 4.1973 & $(-6.2514,18.9324)$ \\
10 & 7.1421 & 4.5108 & $(-6.3903,20.6745)$ \\
11 & 7.4984 & 4.8388 & $(-7.018,22.0148)$ \\
12 & 6.7128 & 4.6415 & $(-7.2117,20.6373)$ \\
13 & 8.4212 & 5.7918 & $(-8.9542,25.7966)$ \\
14 & 10.0199 & 6.0708 & $(-8.1925,28.2323)$ \\
\hline
\end{tabular}

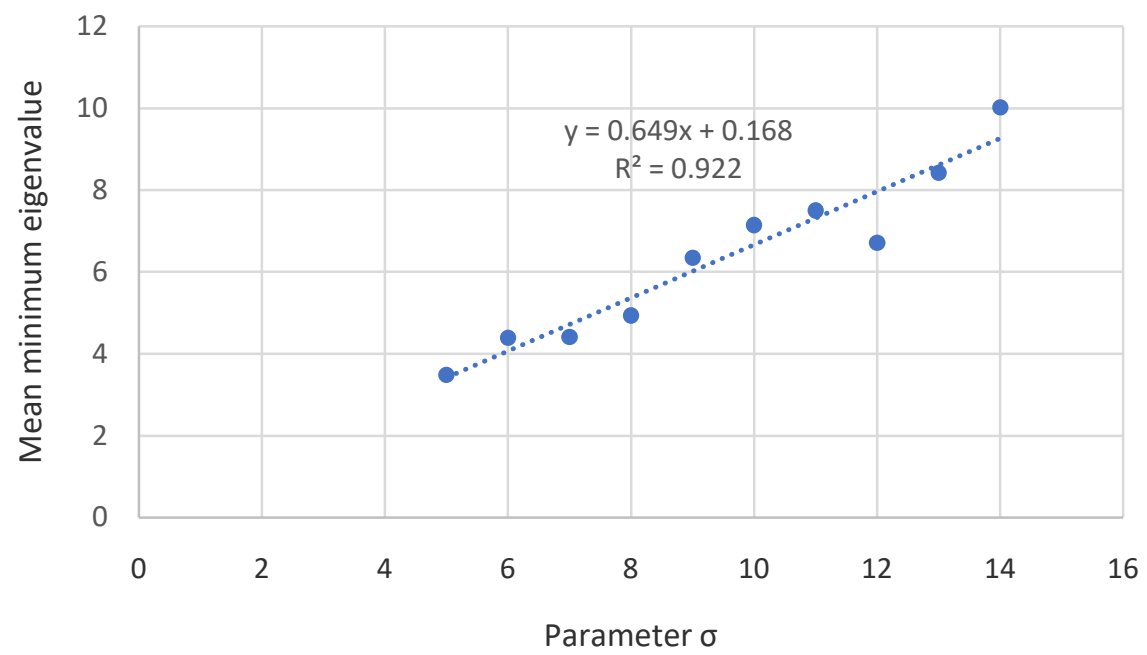

Figure 8. Line of best fit between parameter $\sigma$ and mean minimum eigenvalue of $5 \times 5$ random matrix. 
On comparing Figures 1-2 with corresponding Figures 5-6 and Figures 3-4 with corresponding Figures 7-8, an interesting observation is that by reducing the order of the random matrix, the pattern, or the lack of it, of predicted maximum or minimum eigenvalue of matrix does not significantly vary. But if we look at the corresponding tables, we find that the maximum eigenvalue is reduced by about half in magnitude when the order of the matrix is reduced by half while the minimum eigenvalue remains unaffected. Further, from Figures 1 and 5, Figures 3 and 7, we can propose the following linear model for predicting the maximum eigenvalue (response $\mathrm{y}_{\max }$ ) for a given $\mu$ as predictor and $\varepsilon$ as error component:

$$
\mathrm{y}_{\max }=\beta_{0}+\beta_{1} \mu+\varepsilon
$$

(as there is no dependence on $\sigma$, so $\sigma$ cannot be used as a predictor in the model)

Similarly, from Figures 2 and 6, Figures 4 and 8, we can propose the following linear model for predicting the minimum eigenvalue $\left(\mathrm{y}_{\min }\right.$ response) for a given $\sigma$ as predictor and $\varepsilon^{\prime}$ as error component:

$$
\mathrm{y}_{\min }=\gamma_{0}+\gamma_{1} \sigma+\varepsilon^{\prime}
$$

(as there is no dependence on $\mu$, so $\mu$ cannot be used as a predictor in the model)

The parameters in both the linear models can be obtained using the principle of least squares [7] for simultaneous variation in $\mu$ and $\sigma$.

Our previous studies on predicting maximum and minimum eigenvalues for square random matrices filled with iid variates from different distributions, namely, Binomial, continuous uniform, discrete uniform and exponential distribution have shown that the predictive linear model depends on all the parameters of the input distribution [8]. In contrast, in this paper, we came to know that in case of normal distribution input, the linear model does not depend upon all the parameters.

\section{Theoretical Analysis for Predicting the Range of the Sum of All the Eigenvalues of a Random Matrix}

For any diagonalizable matrix whose entries are random coming from a particular probability distribution (normal in our case), we can predict the range of sum of eigenvalues using trace of the matrix.

We know that, trace of a diagonalizable matrix is equal to sum of its eigenvalues.

i.e., if $\mathrm{A}$ is a diagonalizable square matrix of order nxn then,

Sum of eigenvalues of $\mathrm{A}=\operatorname{tr}(A)=\sum_{i=1}^{n} a_{i i}$

Taking mathematical expectation,

$$
E(\operatorname{tr}(A))=E\left(\sum_{i=1}^{n} a_{\mathrm{ii}}\right)=\sum_{i=1}^{n} E\left(a_{\mathrm{ii}}\right)
$$

assuming that the expectation exists and are finite.

Since, $a_{\mathrm{ii}}$ 's are iid $\mathrm{N}\left(\mu, \sigma^{2}\right)$ variates, hence

$E\left(a_{\mathrm{ii}}\right)=\mu$ and $\operatorname{Var}\left(a_{\mathrm{ii}}\right)=\sigma^{2}$

$E(\operatorname{tr}(\mathrm{A}))=\mathrm{n} \mu$ from $(4)$

$\operatorname{var}(\operatorname{tr}(\mathrm{A}))=\operatorname{Var}\left(\sum_{i=1}^{n} a_{\mathrm{ii}}\right)=\sum_{i=1}^{n}\left(\operatorname{Var}\left(a_{\mathrm{ii}}\right)\right)=\mathrm{n} \sigma^{2}$ (since $a_{\mathrm{ii}}$ 's are independent).

Chebyshev's inequality states that,

$$
P(E(X)-k \times S . D(X)<X<E(X)+k \times S . D(X)) \geq 1-1 / k^{2}
$$

where $S . D$ stands for standard deviation (defined as the positive square root of variance). For a proof of this inequality, see [2]. In our case,

$$
\begin{aligned}
& P\left\{n \mu-k \times \sqrt{ }\left(n \sigma^{2}\right)<\operatorname{tr}(A)<n \mu+k \times \sqrt{ }\left(n \sigma^{2}\right)\right\} \geq 1-1 / k^{2} \\
& P(n \mu-k \times \sigma \sqrt{n}<\operatorname{tr}(A)<n \mu+k \times \sigma \sqrt{n}) \geq 1-1 / k^{2}
\end{aligned}
$$

In this way, we can predict the range of the sum of all the eigenvalues of a diagonalizable $n \times n$ square matrix whose elements are iid variates coming from normal distribution with parameters $\mu$ and $\sigma^{2}$.

Since $\operatorname{tr}(A)=\sum_{\mathrm{i}=1}^{\mathrm{n}} \mathrm{a}_{\mathrm{ii}}$ being a linear combination of independent normal variates is itself a normal variate, we could use a tighter result $\mathrm{P}(|\mathrm{Z}|<1.96)=0.95$

where $\mathrm{Z}=[\operatorname{tr}(\mathrm{A})-\mathrm{E}\{\operatorname{tr}(\mathrm{A})\}] / \mathrm{SD}\{\operatorname{tr}(\mathrm{A})\}$ is a standard normal variate.

This yields $\mathrm{P}(\mathrm{n} \mu-1.96 \sigma \sqrt{ } \mathrm{n}<\operatorname{tr}(\mathrm{A})<\mathrm{n} \mu+1.96 \sigma \sqrt{ } \mathrm{n})=0.95$.

The only reason for using Chebyshev's inequality even for the normal case is to make our results comparable with those for other distributions where such a tighter result as given above may not be available. 


\section{Conclusion}

It appears that it is quite possible to predict the maximum and minimum eigenvalues as well as the range of the sum of eigenvalues of a random matrix in terms of the parameter(s) of the probability distribution constituting the matrix elements using a linear model. Our paper, demonstrates how this can be done citing normal distribution as a case study. It also appears that while the order of the matrix affects the sum of the eigenvalues, it has interestingly no significant effect on the magnitude of the minimum eigenvalue while that of the maximum eigenvalue is reduced by half. The most important finding is that in case of normal distribution input, the maximum or minimum eigenvalue is not depending on both the parameters of the input distribution but only one of them. In earlier studies such dependence was on all the parameters of the input distribution.

\section{Ethical Declaration}

The authors hereby declare that this research does not receive any funding. They further declare that they have no conflict of interest.

\section{References}

[1] Hoffman, K. and Kunze, R. (1971). Linear Algebra, Prentice-Hall Inc. Eaglewood Cliffs, New Jersey.

[2] Gupta, S. C. and Kapoor, V. K. (2014). Fundamentals of Mathematical Statistics, Sultan Chand \& Sons.

[3] Kennedy, W. J. and Gentle, J. E. (1980). Statistical Computing, Marcel Dekker Inc.

[4] Girko, V. L. (1985). Spectral theory of random matrices, The British Library and The London Mathematical Society.

[5] Majumdar, S. N., and Schehr, G. (2014). Top eigenvalues of a random matrix: Large deviations and third order phase transition, IOP Publishing Ltd \& SISSA Medialab sri.

[6] Liu, Yi-kai. (2001). Statistical Behaviour of the Eigenvalue of the Random Matrices. http://web.math.princeton. edu/mathlab/projects/ranmatrices/yl/randmtx. PDF accessed on June 1, 2020.

[7] Draper, N. and Smith, H. (1998). Applied Regression Analysis. Wiley, $3^{\text {rd }}$ ed.

[8] Princila and Chakraborty, S. (2020). Predicting Maximum and Minimum Eigenvalues of a Random Matrix: A Study in Simulation. Rathore Academic Research Publications. 